\title{
Rupture of a Giant Posterior Inferior Cerebellar Artery Aneurysm in an Infant Following a Ventriculoperitoneal Shunt
}

\author{
-Case Report-
}

\author{
Ketan Desai, Trimurti NadKarni, Dattatraya Muzumdar, and Atul GOEL
}

Department of Neurosurgery, King Edward Memorial Hospital, Seth G. S. Medical College, Parel, Mumbai, India

\begin{abstract}
A 4-month-old female child presented with a huge posterior fossa mass lesion and severe hydrocephalus. Six hours after a ventriculoperitoneal shunt procedure, her condition worsened and she died. Autopsy showed extensive intracranial hemorrhage due to rupture of a posterior inferior cerebellar artery aneurysm. The probable causes of the rupture of the aneurysm were mechanical effects or changes in cerebral blood flow.
\end{abstract}

Key words: giant aneurysm, hydrocephalus, ventriculoperitoneal shunt, pediatric patients

\section{Introduction}

Intracranial aneurysms are seldom encountered in the pediatric age group, and those within the posterior circulation are even more rare.1,10,17,26,28) Pediatric and adult aneurysms show several differences, but the evaluation and management is similar. We present a case of massive posterior inferior cerebellar artery (PICA) aneurysm in an infant manifesting as symptoms of a mass lesion, marked hydrocephalus, and aneurysm rupture following a shunt operation.

\section{Case Report}

A 4-month-old female child presented with progressive increase in the size of the head and delayed development milestones. The child was born by cesarean delivery because of large head size at birth. On admission, she had macrocephaly with a head circumference of $48 \mathrm{~cm}$. The anterior fontanel was tense and bulging. The scalp veins were distended and tortuous. The child was irritable and had upward gaze palsy. Computed tomography of the brain showed a massive, isodense, highly enhanced mass

Received June 21, 2000; Accepted November 9, 2000 occupying almost the whole posterior cranial fossa (Fig. 1). There was obstructive hydrocephalus and marked dilation of the third and lateral ventricles. There was no evidence of subarachnoid hemorrhage (SAH) (Fig. 2). Due to the symptoms and radiological features, a right ventriculoperitoneal shunt was performed, before considering angiography. The ventricular catheter was passed through a parietal burr hole and fitted with a standard medium discharge valve. The cerebrospinal fluid (CSF) was clear and colorless and under high pressure. The child was drowsy after the surgery. She died suddenly about 6 hours later following cardiorespiratory arrest. Autopsy showed extensive SAH and intraventricular hemorrhage secondary to rupture of a relatively thick-walled giant aneurysm arising at the origin of the left PICA from the vertebral artery. The aneurysm had ruptured at the fundus. The surrounding brainstem and cerebellum were severely distorted and compressed. Ventriculomegaly was obvious and the shunt catheter was noted to be in the optimum position. No brainstem hemorrhages were seen.

\section{Discussion}

Intracranial aneurysms in the pediatric age group are uncommon, accounting for about $0.5-4.6 \%$ of all 


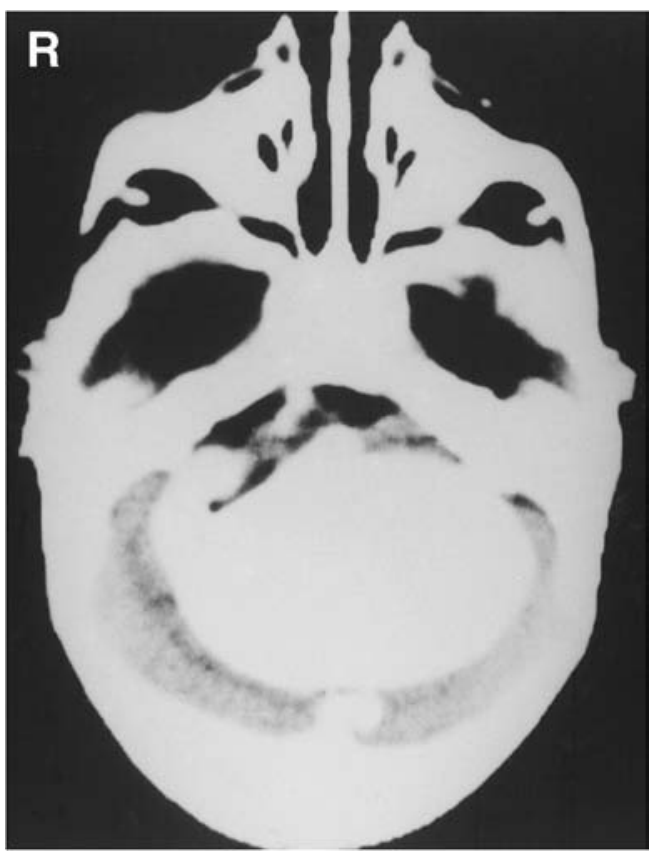

Fig. 1 Axial computed tomography scan with contrast medium showing a large, highly enhanced mass in the posterior cranial fossa.

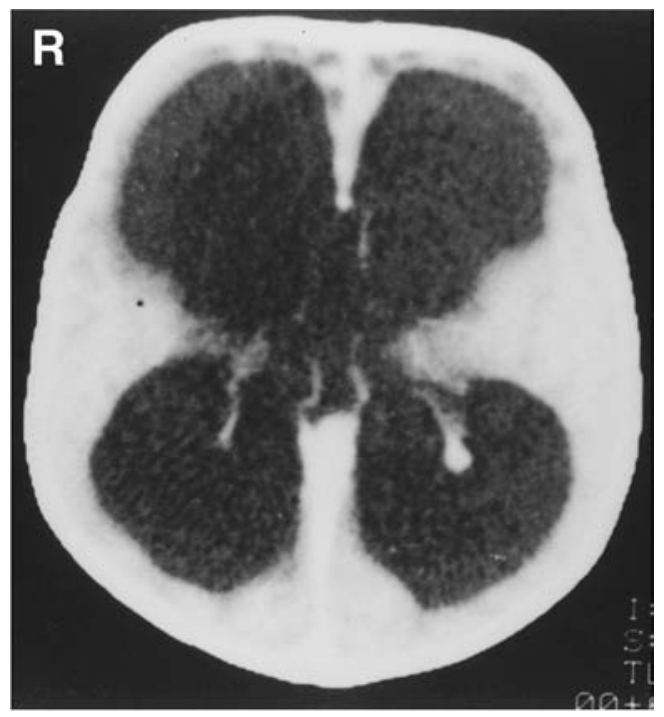

Fig. 2 Axial computed tomography scan showing marked dilation of the lateral and third ventricles.

intracranial aneurysms. ${ }^{1,10,17,23,26)}$ A large co-operative study reported only one patient with aneurysm under the age of 4 years among 6368 cases. ${ }^{15)} \mathrm{An}$ autopsy study of over 8000 brains of children failed to find a single intracranial aneurysm. ${ }^{12)}$ Pediatric aneurysms distinctly differ from adult aneurysms in respect to male predominance $(2: 1$ to $3: 1)$, and disproportionately high incidences of posterior circulation aneurysms $(40-50 \%)$ and of giant aneurysms $(30-45 \%){ }^{18-20)}$ Aneurysms of the PICA are extremely rare in the pediatric age group..$^{3,11,13,21,27)}$

The aneurysm in our patient was so huge that it occupied almost the entire posterior cranial fossa. No previous case of such a large aneurysm has been reported. The commonest presentation of intracranial aneurysm is SAH. Incidence of tumor-like presentation is significantly higher in early childhood due to the prevalence of giant aneurysms in this age group., ${ }^{2,2023}$ Giant posterior cranial fossa aneurysms present with cranial nerve deficits, brainstem dysfunction, or hydrocephalus. ${ }^{20,23)}$ In the co-operative study, only $0.2 \%$ of the patients with aneurysmal SAH presented in the first decade of life. ${ }^{15)}$ Not many years ago, aneurysms of the posterior circulation were only a postmortem curiosity, because of the difficulty in diagnosing posterior cranial fossa aneurysms which had not bled. ${ }^{16)}$

Giant aneurysms are relatively tough walled and bleeding secondary to CSF drainage has never been reported. Rupture of the more typical berry aneurysm following drainage through lumbar puncture has been frequently recorded. ${ }^{822)}$ Release of the tamponade effect due to the drainage of CSF may be the cause of the rupture. The clinical features in our case suggested that decompression of ventricles as a result of the shunt operation was important in the rupture of the aneurysm.

Preoperative shunt surgery, temporary drainage of CSF, or diversion of CSF flow is advised in various situations. This procedure is intended to reduce the raised intracranial pressure and relieve the patient's symptoms, to normalize the altered cerebral blood flow, and to 'relax' the brain, which will assist the surgeon in the definitive surgical procedure on the tumor. Hemorrhages in cranial tumors, impaction of spinal tumors and resultant neurological deficits, and SAH following drainage of either ventricular or lumbar CSF have been frequently reported. Such effects could be due to movements in the lesions secondary to changes in the critically balanced intracranial or intraspinal pressure mechanism. ${ }^{14,24,25)}$ Hydrocephalus could be a protective phenomenon of the brain in cases of tumors and obstructive hydrocephalus. ${ }^{5}$ ) Supratumoral raised intracranial pressure could be a mechanism whereby the tumor is pushed away from the vital neural structures like the brainstem and hypothalamus. ${ }^{6,7)}$

Movement of the giant aneurysm following shunt 
surgery in our case could have resulted in shearing strain and consequent rupture. Another possible mechanism is an increase in cerebral blood flow following ventriculostomy and a sudden decrease in the intracranial pressure which the aneurysm wall could not tolerate. ${ }^{4,9)}$ The rebleeding rate is less for aneurysms in children, whereas others have reported rebleeding rates ranging from $13-33 \% .^{19,23)}$ Although vasospasm occurs in children, the incidence of ischemic deficits is relatively low. The overall prognosis for children with aneurysmal SAH is better than for adults. ${ }^{9,11)}$

The occurrence of a giant PICA aneurysm in a 4-month-old female infant is rare. The ventriculoperitoneal shunt procedure precipitated a fatal SAH. The prognosis for such infants is poor. Huge posterior fossa aneurysm should be considered in evaluating patients with infratentorial masses. Magnetic resonance (MR) imaging with MR angiography or computed tomography angiography could better delineate such a lesion. Endovascular therapy or surgery can then be contemplated.

\section{References}

1) Allison JW, Davis PC, Sato Y, James CA, Haque SS, Angtuaco EJ, Glasier CM: Intracranial aneurysms in infants and children. Pediatr Radiol 28: 223-229, 1998

2) Amacher AL, Drake CG: The results of operating upon cerebral aneurysms and angiomas in children and adolescents. I. Cerebral aneurysms. Childs Brain 5: 151-165, 1979

3) Eben A, Davis DH, Pikula L: Aneurysm of the posterior inferior cerebellar artery filling the fourth ventricle. J Neurosurg 24: 99-101, 1966

4) Furguson GG: Turbulence in human intracranial saccular aneurysms. J Neurosurg 33: 485-497, 1970

5) Goel A: Whither preoperative shunts for posterior fossa tumors? Br J Neurosurg 7: 395-399, 1993

6) Goel A: Preoperative shunts in suprasellar tumors. $\mathrm{Br}$ J Neurosurg 9: 189-193, 1995

7) Goel A, Nitta J, Kobayashi S: Tumor obstructive hydrocephalus: a natural defense mechanism, in Kobayashi S, Goel A, Hongo K (eds): Neurosurgery of Complex Tumors and Vascular Lesions. New York, Churchill Livingstone, 1997, pp 373-391

8) Hart IK, Bone I, Hadley DM: Development of neurological problems after lumbar puncture. Br Med J 296: 51-52, 1988

9) Hartman A, Alberti E, Lange D: Effects of CSF drainage on CBF and CBV in subarachnoid hemorrhage and communicating hydrocephalus. Acta Neurol Scand Suppl 64: 336-337, 1977

10) Herman JM, Rekate HL, Spetzler RF: Pediatric intracranial aneurysms: simple and complex cases. Pediatr Neurosurg 17: 66-72, 1991
11) Hosotani K, Tokuriki Y, Takebe Y, Kawaguchi K, Tsuji A, Kubota T: Ruptured aneurysm of the distal PICA in a neonate: case report. Neurol Med Chir (Tokyo) 35: 892-895, 1995

12) Housepian EM, Pool JL: A systemic analysis of intracranial aneurysms from autopsy file of Presbyterian Hospital. J Neuropathol Exp Neurol 17: 409-423, 1958

13) Jane JA: A large aneurysm of the posterior inferior cerebellar artery in a 1-year-old child. J Neurosurg 18: 245-247, 1965

14) Koshu K, Tominaga T, Fujii Y, Yoshimoto T: Quadriparesis after a shunting procedure in a case of cervical spinal neurinoma associated with hydrocephalus: case report. Neurosurgery 32: 669-670, 1993

15) Locksley HB: Report on the cooperative study of intracranial aneurysms and subarachnoid hemorrhage. Section V, part 1. Natural history of subarachnoid hemorrhage, intracranial aneurysms and arteriovenous malformations. Based on 6368 cases in the cooperative study. J Neurosurg 25: 219-239, 1966

16) Michael WF: Posterior fossa aneurysms simulating tumors. J Neurol Neurosurg Psychiatry 37: 218-223, 1974

17) Norris JS, Wallace MC: Pediatric intracranial aneurysms. Neurosurg Clin N Am 9: 557-563, 1998

18) Osenbach RK: Giant aneurysm of the distal posterior inferior cerebellar artery in an 11-month-old child presenting with obstructive hydrocephalus. Pediatr Neurosci 15: 309-312, 1989

19) Ostergaard JR, Voldby B: Intracranial arterial aneurysms in children and adolescents. J Neurosurg 58: 832-837, 1983

20) Patel AN, Richardson AE: Ruptured intracranial aneurysms in the first two decades of life. J Neurosurg 35: 571-576, 1971

21) Pickering LK, Hogan GR, Gilbert EF: Aneurysm of the posterior inferior cerebellar artery rupture in newborn. Am J Dis Child 119: 155-158, 1970

22) Profeta G, de Falco R, Profeta L: [Reasons against lumbar puncture diagnosis with cerebrospinal fluid drainage in subarachnoid hemorrhage]. Minerva Anestesiol 64: 137-139, 1998 (Ital, with Eng abstract)

23) Storrs BB, Humphreys RP, Hendrick EB, Hoffman $\mathrm{HJ}$ : Intracranial aneurysms in the pediatric agegroup. Childs Brain 9: 358-361, 1982

24) Vaquero J, Cabezudo JM, DeSola RG, Nombela L: Intratumoral hemorrhage in posterior fossa tumor after ventricular drainage. Report of two cases. J Neurosurg 54: 406-408, 1981

25) Wakai S, Yamakawa K, Manaka S, Takakura K: Spontaneous intracranial hemorrhage caused by brain tumor: its incidence and clinical significance. Neurosurgery 10: 437-444, 1982

26) Yazbak PA, McComb JG, Raffel C: Pediatric traumatic intracranial aneurysms. Pediatr Neurosurg 22: 15-19, 1995

27) Yeh HS, Tomsick TA, Tew JM: Intraventricular hemorrhage due to aneurysms of the posterior inferior cerebellar artery. J Neurosurg 62: 772-775, 
1985

28) Zingale A, Chiaramonte I, Consoli V, Albanese V: Distal posterior inferior cerebellar artery saccular and giant aneurysms: report of two new cases and a comprehensive review of the surgically treated cases. J Neurosurg Sci 38: 93-104, 1994
Address reprint requests to: T. Nadkarni, M.D., Noor Mansion, Ground Floor, Chowpatty Sea Face, Mumbai 400 007, India. 